\title{
From Mathematical Physics to Analysis: A Walk in Barry Simon's Mathematical Garden
}

Editor's Note: Fritz Gesztesy kindly accepted our invitation to put together this feature in honor of Barry Simon on the occasion of Simon's 2016 AMS Leroy P. Steele Prize for Lifetime Achievement and his 70th birthday conference this August 28-September 1.

\section{Fritz Gesztesy}

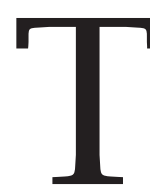

his is a collection of contributions by collaborators, postdocs, and students of Barry Simon of the California Institute of Technology on the occasion of Simon's receiving the 2016 AMS

Leroy P. Steele Prize for Lifetime Achievement. The citation for the award mentions his tremendous impact on the education and research of a whole generation of mathematical scientists, and we will underscore this by demonstrating his penetrating influence on topics ranging from quantum field theory, statistical mechanics, the general theory of Schrödinger operators, spectral and inverse spectral theory to orthogonal polynomials.

But we should start at the beginning: Barry was born to parents Minnie and Hy Simon in 1946, and together with his older brother, Rick, grew up in Brooklyn, New York. There he attended James Madison High School, obtaining a perfect score on the MAA's American High School Mathematics Examinaton in 1962 and thus becoming the subject of an article in the New York Times at the tender age of sixteen. Under the influence of Sam Marantz, an inspiring physics teacher in high school, he applied to Harvard and was admitted. While at Harvard he was a top five Putnam Competition Winner in 1965 and received his BA summa cum laude in physics in 1966. George Mackey at Harvard recommended Barry pursue a doctorate with Arthur Wightman at Princeton because Wightman was well known for advocating the application of rigorous mathematics in physics.

Fritz Gesztesy is Mahala and Rose Houchins Professor of Mathematics at the University of Missouri, Columbia. His email address is gesztesyf@missouri.edu. Since August 1 he is Jean and Ralph Storm Chair of Mathematics at Baylor University. His email address there is Fritz_Gesztesy@baylor. edu.

For permission to reprint this article, please contact:

reprint-permission@ams.org.

DOI: http://dx.doi.org/10.1090/noti1403

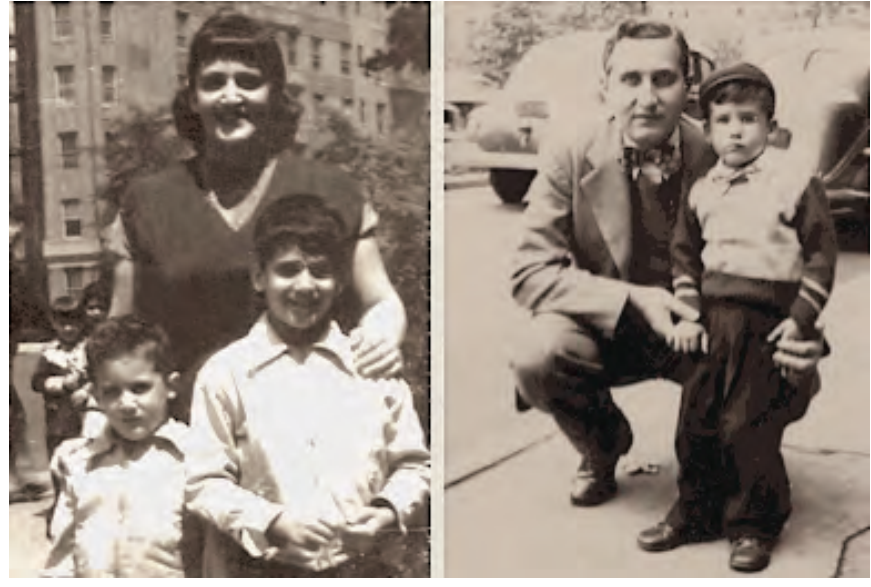

Barry Simon with his mother and brother, Rick (left), and with his father (right) (ca. 1950).

Barry Simon completed his doctorate in physics at Princeton under Wightman's supervision in 1970. The body of his work during the time of his doctoral research was of such importance that he was immediately appointed to assistant professor, jointly in mathematics and physics, at Princeton. He rapidly rose to the rank of full professor by 1976. Several contributions below will attest to the electric atmosphere at Princeton in those days, making it a thriving center for quantum field theory, statistical mechanics, and nonrelativistic quantum mechanics. Barry joined Caltech in 1981, holding the position of IBM Professor of Mathematics and Theoretical Physics since 1984. At Caltech, Barry's interests further broadened into areas such as random and ergodic Schrödinger operators, exotic spectra, inverse spectral theory, and the analytic theory of orthogonal polynomials. 


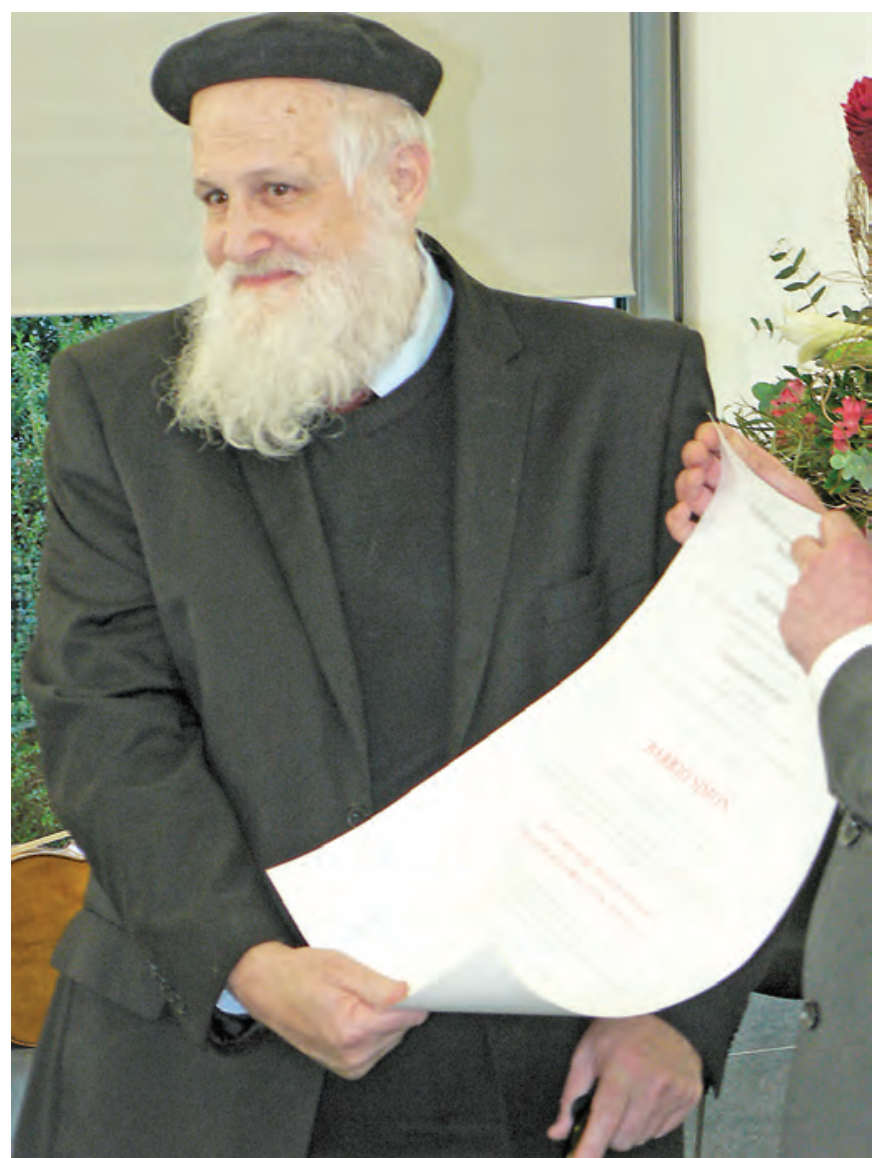

Honorary doctorate, Ludwig-Maximilians-University of Munich, 2014.

Since many of Barry's major research accomplishments are discussed in depth and put into proper context in the vari-

... rigorous mathematics in physics ous contributions to follow, we itemize only a few brief comments at this point, focusing on some key results he proved, fundamental concepts he advocated, and some of the important terms he and his collaborators first coined with lasting impact:

- a rigorous framework for resonances, complex and exterior scaling, Fermi's golden rule, proof of the Oppenheimer formula for the Stark effect, convergence of time-dependent perturbation theory;

- constructive (Euclidean) quantum field theory in two space-time dimensions, connections to statistical mechanics, lattice approximations and correlation inequalities, $P(\phi)_{2}$ spatially cutoff field theories;

- hypercontractive and ultracontractive semigroups;

- magnetic fields, diamagnetic inequality, Kato's inequality;
- a proof of continuous symmetry breaking in classical and quantum statistical models;

- Thomas-Fermi theory, semiclassical bounds, nonWeyl asymptotics;

- asymptotic perturbation theory of eigenvalues: Borel and Padé summability, Zeeman effect, anharmonic oscillators, instanton tunneling, BirmanSchwinger principle, coupling constant thresholds;

- general theory of Schrödinger operators: essential self-adjointness, pointwise bounds on eigenfunctions, path integral techniques, absence of singular continuous spectrum in $N$-body sytems;

- Berry's phase and holonomy, homotopic interpretation of the Thouless integers and topological structure in the integer quantum Hall effect;

- random and almost periodic Schrödinger and Jacobi operators, exotic spectral phenomena (Cantor, singular continuous, and dense pure point spectra) and their transition to becoming a central object in mathematical physics (the singular continuous revolution), Wonderland theorem, Thouless formula, almost Mathieu equation;

- trace formulas for potential coefficients in terms of the Krein-Lifshitz spectral shift function, uniqueness theorems in inverse spectral theory for Schrödinger and Jacobi operators, oscillation theory in gaps of the essential spectrum, inverse spectral analysis with partial information on the potential;

- a new approach (the analog of the continued fraction method) to inverse spectral theory of Schrödinger operators, his local Borg-Marchenko theorem;

- a systematic application of operator theory techniques to orthogonal polynomials on the real line (OPRL) and on the unit circle (OPUC), CMV matrices, Verblunsky coefficients;

- sum rules for Jacobi matrices and applications to spectral theory (Killip-Simon theorem), perturbations of OPRL and OPUC with periodic recursion coefficients;

- Szegó asymptotics, a proof of Nevai's conjecture and its finite gap extension, the finite gap analog of the Szegó-Shohat-Nevai theorem, the fine structure of zeros of orthogonal polynomials (clock behavior), higher-order Szegő theorems.

Barry Simon's influence on our community by far transcends his approximately four hundred papers, particularly in view of 126 coauthors, 50 mentees, 31 graduate students, and about 50 postdocs mentored. In this context, one must especially mention his twenty books, the first fifteen of which have educated scores of mathematicians and mathematical physicists, two generations by now, and continuing into the foreseeable future.

One cannot overestimate the influence of Reed and Simon's four-volume series, Methods of Modern Mathematical Physics, I-IV (1972-79). It took on the same level of importance that Courant-Hilbert's two volumes had for previous generations, and it continues to fill that role 
all over the globe to this day. To gauge the importance of Reed and Simon behind the Iron Curtain, we contacted Albrecht Böttcher (TU Chemnitz, Germany). Like so many in our generation, Albrecht has a very personal relationship with Volume I and underscored (his words) "the truly ingenious selection and presentation of the mathematical topics." The latter sentiment, however, is by no means unique to colleagues who read Reed-Simon in Russian translation; it is just as prevalent in the West.

Cumulative sales figures for all four volumes to date top 37,000 copies. Barry's other books, most notably Functional Integration and Quantum Physics (1979), Trace Ideals and Their Applications (1979, 2005), Orthogonal Polynomials on the Unit Circle, Parts 1, 2 (2005), and Szegó's Theorem and Its Descendants (2011), profoundly influenced research in these areas. Finally, we have not even begun to understand the legacy which will be created with his newest five-volume set, A Comprehensive Course in Analysis (2015), which offers a panorama from real to complex and harmonic analysis all the way to operator theory.

In short, Barry has been a phenomenal force in mathematical physics, encyclopedic in his knowledge and a grand master of mathematical structure and abstract analysis. Above all, he truly enjoys collaboration and the human interactions that come with it. As a sign of his tremendous influence on our community we note that to date MathSciNet lists 15,325 citations by 6,602 authors, and Google Scholar lists 61,680 citations and an h-index of 104.

Barry has been recognized with honorary degrees from the Technion, Haifa; the University of Wales-Swansea, and LMU-Munich. He was awarded the Stampacchia Prize in 1982 with M. Aizenman, the Poincaré Prize of the International Association of Mathematical Physics in 2012,

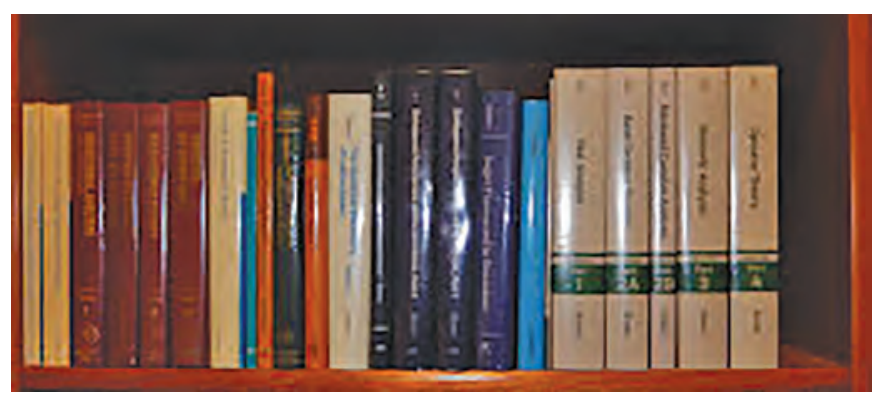

The books Barry Simon has authored ...thus far. and the Bolyai Prize of the Hungarian Academy of Sciences in 2015. He is a fellow of the American Physical Society (1981), the American Academy of Arts and Sciences (2005), and the AMS (2013). He has served as vice president of the AMS and of IAMP.

Finally, on a personal note: Having been a frequent collaborator of Barry's, I have often been approached with the assumption of being one of Barry's students or postdocs, but this is not the case. On the other hand, like so many of my generation, I learned about the tools of our trade through his writings and especially from Reed and Simon I-IV, so of course it feels like I was Barry's student, even though the proper term appears to be that I'm one of his many mentees. Barry has been a constant inspiration to me for about forty years now; I feel incredibly fortunate that he became my mentor and friend.

\section{Evans M. Harrell}

\section{Singular Perturbation Theory and Resonances}

The very first article in Barry Simon's publication list, which appeared in Il Nuovo Cimento when he was a 22year-old graduate student, was concerned with singular perturbation theory. This paper showed that a certain regularized, renormalized perturbation expansion for a two-dimensional quantum field theory model converges with a positive radius of convergence. As Barry candidly admitted in that article, in itself the result was of limited significance, but in a subject for which at that time "all the mathematically suitable results...are of a negative nature," it announced a new, more constructive era.

To the reader familiar with Barry Simon's works on mathematical physics of the 1970s, it is striking how many of the hallmarks of his technique are already apparent in this first article. Before entering deeply into the research, Barry first carried out a thorough and penetrating review of the entire literature on the subject. This signature of his method was something those of us who were students at Princeton in the 1970 s would witness every time Barry began a new research project: Seeing him emerge from the library shared by Jadwin and Fine Halls with a mountain of books and articles, it was humbling to realize that Barry was not merely brave enough to collect all of the knowledge about the next subject he wished to study, but seemingly overnight he would absorb it in detail and carefully assess each contribution for its mathematical appropriateness. True to form, in that first article, Barry laid out which claims in the literature were established with mathematical rigor, which were plausibly to be believed, perhaps with some extra attention to assumptions, and which were frankly dubious. Finally, Barry's own way of formulating the problem was sparse and clear, and his reasoning incisive.

The perturbation theory that applies to nonrelativistic quantum mechanics is a linear theory, allowing straightforward calculations of systematically corrected eigenvalues

Evans M. Harrell is professor emeritus at the School of Mathematics at the Georgia Institute of Technology. His email address is harre11@math.gatech.edu. 


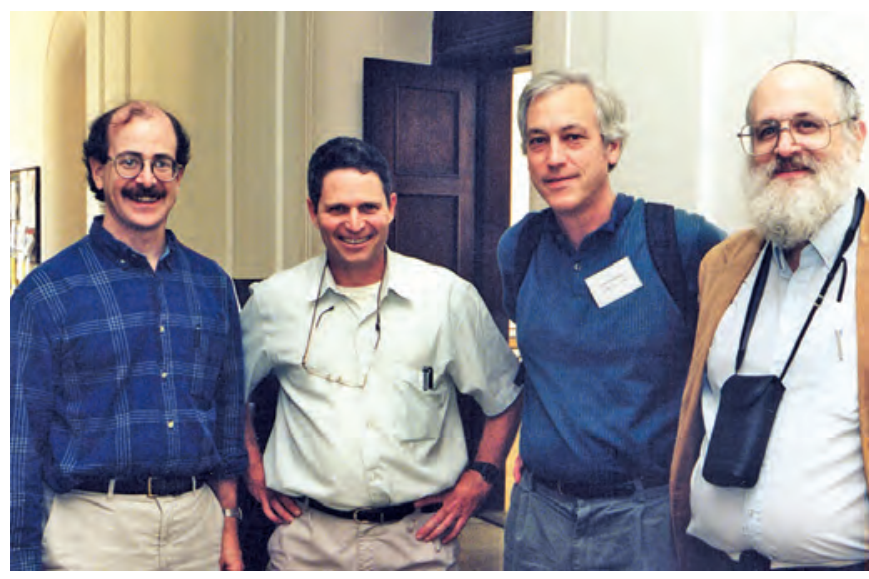

Lorenzo Sadun, Yosi Avron, Evans Harrell, Barry Simon (ca. 1988).

and eigenfunctions. Schrödinger's adaptation of the procedures of Rayleigh to calculate the shifts in hydrogen emission spectrum in the presence of an electric field (known as the Stark effect) went a long way in establishing the validity of his new quantum theory. This success is ironic, given that the series for which Schrödinger and Epstein calculated the first terms has radius of convergence zero, and the spectrum of the Stark Hamiltonian for any nonzero value of the electric field is purely continuous, containing no discrete eigenvalues at all. The instant the interaction is switched on, the nature of the spectrum changes radically, and the perturbed eigenvalue becomes a resonance state! The Stark effect is known today to belong to the realm of singular perturbation theory.

In the two decades after Schrödinger's work, Some mathematicians created a comprehensive theory of linear physicists were "using methods of unknown validity" operators on Hilbert space. In the 1930s and 1940s Rellich and Kato produced a mathematically rigorous theory of regular perturbations of linear operators and some aspects of singular perturbations. In their hands, perturbation theory was concerned with analytic operator-valued functions of a complex variable, defined initially as convergent power series with operator coefficients, which are most typically self-adjoint in applications to quantum mechanics. These functions exhibit the range of behavior of ordinary scalar analytic functions of a complex variable, with manageable complications when the spectrum consists of discrete eigenvalues of finite multiplicity, and some new phenomena when there is an essential spectrum. Physicists and chemists at this time solved problems and developed perturbative techniques that sometimes fell into the domain of regular perturbation, but just as frequently produced series that could be calculated term by term while exhibiting singular features, as in a

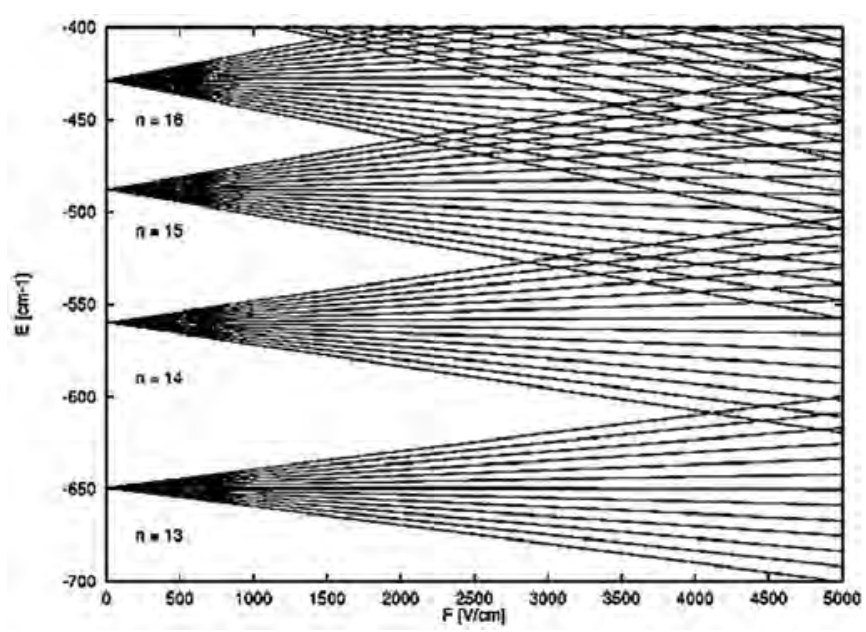

The Stark effect: The spectrum of the hydrogen atom under an increasingly intense electric field, from the Courtney-Spellmeyer-Jiao-Kleppner article appearing in Physical Review A, vol. 51 (1995).

zero radius of convergence or, worse, convergence but to the wrong answer. (For instance, a resonance eigenvalue associated with tunneling may have a nonzero imaginary part that, typically, is represented in perturbation theory by a series of the form $\sum_{n} c_{n} \beta^{n}$ with $c_{n}=0$ for all $n$.)

At the time Barry Simon hit the scene, an industry was thriving in attempts to get information from such expansions, whether by replacing the series by other expressions, especially Padé approximates $P[m, n]$, which are ratios of polynomials of $m$ and $n$ degrees, or by the use of analytic-function techniques like Borel summability to make sense of divergent series. Many of Barry's early works addressed these topics.

One of the important models in quantum mechanics with a singular perturbation is the quartic anharmonic oscillator. Its Hamiltonian is

$$
p^{2}+x^{2}+\beta x^{4},
$$

and it was the subject of a landmark study by Bender and Wu in 1968-69 in which, "using methods of unknown validity" - in Barry's memorable phrase-they painted a fascinating and largely correct picture of the analytic structure of the eigenvalues of the anharmonic oscillator as functions of the coupling constant $\beta$, considered as a complex variable. Among other things, Bender and $\mathrm{Wu}$ conjectured that the power series expansion for the ground-state eigenvalue of (1) had radius of convergence 0 . Then-new computational capabilities in symbolic algebra had allowed Bender and Wu and others to calculate perturbation series at high orders, and with the first seventy-five coefficients $a_{n}$ for the ground-state eigenvalue in hand, Bender and Wu specifically conjectured that

$$
a_{n} \sim \pi^{-\frac{3}{2}} \sqrt{6} 3^{n} \Gamma\left(n+\frac{1}{2}\right) .
$$

Barry's response to the explorations of Bender and $\mathrm{Wu}$ was to pen the definitive rigorous analysis of the analytic 


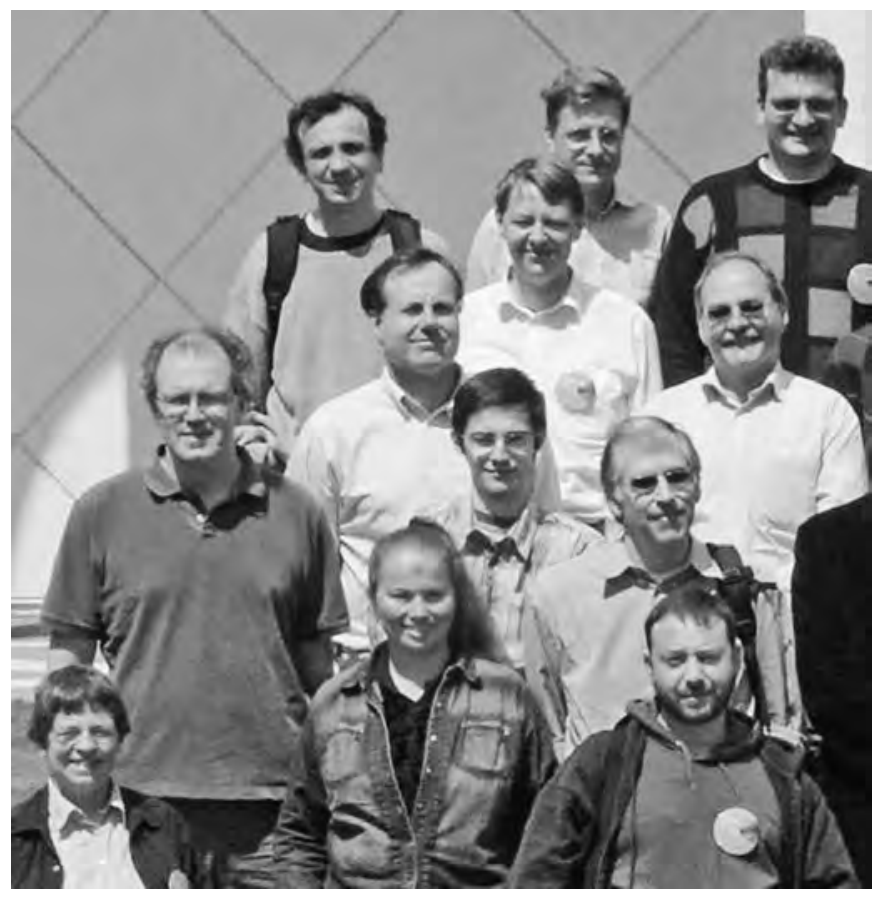

Some of Barry's coauthors, SimonFest 2006, on the occasion of Barry Simon's sixtieth birthday.

properties of the anharmonic oscillator in an extended article in the Annals of Physics in 1970. This work is a timeless classic, a textbook model for how to do singular perturbation theory, and it remains one of Barry's most highly cited works. Most of the claims of Bender and $\mathrm{Wu}$ were put on a firm footing, and many further facts were established. For example, it was shown that $\beta=0$ is a third-order branch point and an accumulation point of singularities. Moreover, Barry obtained sufficient control on the growth rate of perturbation coefficients to show that both the Padé and Borel methods were valid to determine the eigenvalues for nonzero values of $\beta$. A few years later, with the aid of a dispersion relation derived using this understanding of the Riemann surfaces of the eigenvalues, Barry and coauthors proved the formula (2).

Later, several other models of singular perturbation theory, including the Stark and Zeeman effects, received similar treatments in the hands of Barry and his associates, especially Yosi Avron and Ira Herbst. These gems provided a foundation for developments in singular perturbation theory and deepened the understanding of many of the touchstones of quantum mechanics.

Barry has always been quick to recognize others' good ideas when they appear and generous in promoting them in the community of mathematical physicists and beyond. A very pretty method introduced in that era by Aguilar, Balslev, and Combes in 1971 came to be called complex scaling. The original version made use of the dilatation symmetry and complex analysis to move the essential spectrum of a Schrödinger operator into the complex plane, while leaving discrete eigenvalues unaffected. The unitary group of dilatations can be defined via

$$
[U(\theta) f](\mathbf{x}):=e^{v \theta / 2} f\left(e^{\theta} \mathbf{x}\right)
$$

in terms of a real parameter $\theta$. If the scaled potential energy depends in an analytic way on $\theta$, then the parameter can be complexified, and it is easy to see that with a compactness condition on the potential energy the essential spectrum of the complex-scaled Laplacian for nonreal $\theta$ is simply rotated in the complex plane. Meanwhile, isolated eigenvalues are analytic as functions of $\theta$, but since they are constant for real variations in $\theta$, by unique continuation they are also constant for any variation of $\theta$, except that they can appear or disappear when they collide with the essential spectrum, at which point analytic perturbation theory ceases to apply.

With this procedure the ad hoc tradition in physics of treating resonances as nonreal eigenvalues somehow associated with a self-adjoint Hamiltonian became mathematically solid and canonical. I cannot do better in describing complex scaling further than to point to Chapter XIII.10 of Reed-Simon, to which the reader is referred for further details and context. Barry assiduously promoted this excellent tool for understanding resonances, evangelizing the technique to physicists and chemists, by whom it was adopted and used in realistic problems.

I recall in particular when Barry took a delegation of mathematical physicists to the 1978 Sanibel Workshop on Complex Scaling, organized by the noted quantum chemist Per-Olof Löwdin, at which the discussions between the chemists and the believers in mathematical methods "of known validity" were quite fruitful and informative on all sides. Of course, Barry not only recognized, clarified, and promoted the idea of complex scaling but made his own fundamental advances in the subject, especially by greatly extending the set of problems to which it applied by his discovery that it suffices to perform complex scaling on an exterior region.

A similar tale could be told of Barry's recognition of the importance of the microlocal analysis of tunneling phenomena by Helffer and Sjöstrand in the 1980s, which Barry again promoted, clarified, and in certain ways transformed. But space here is limited, and besides, for the reader interested in learning more about singular perturbation theory and resonances, there is an excellent 1991 review article entitled "Fifty years of eigenvalue perturbation theory," written by a master of the genre, Barry Simon himself.

\section{Percy A. Deift}

\section{Princeton in the 1970s; Exponential Decay of Eigenfunctions and Scattering Theory}

The 1970s were a very special time for mathematical physics at Princeton. One can read a lively account of those days, written by Barry himself, in the July 2012 edition of the Bulletin of the International Association of Mathematical Physics. The main thrust of the activity

Percy A. Deift is Silver Professor of Mathematics at the Courant Institute, NYU. His email address is deift@cims.nyu.edu. 


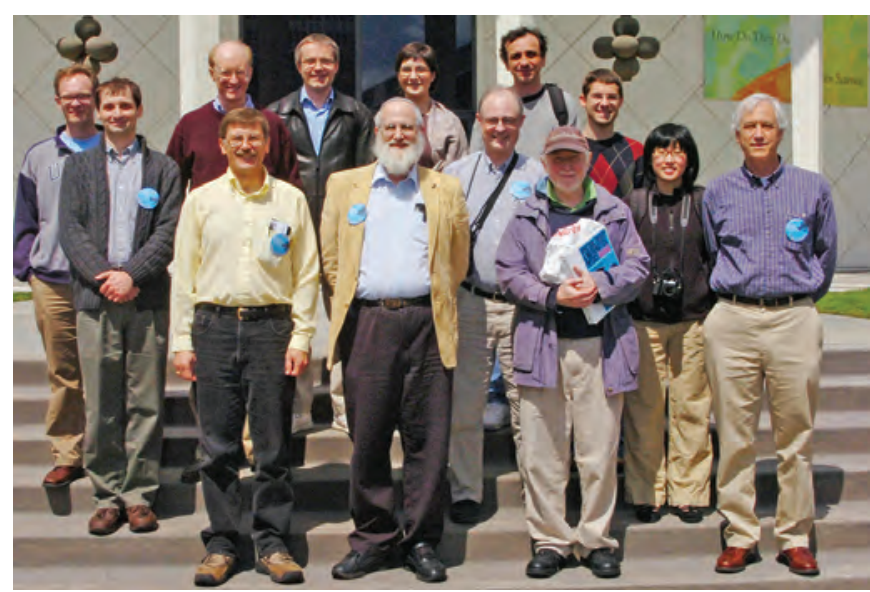

Some of Barry Simon's students, SimonFest 2006, on the occasion of Barry Simon's sixtieth birthday.

was in statistical mechanics, quantum field theory, and nonrelativistic quantum mechanics. The list of people who participated in math-phys at Princeton University in those years as students, postdocs, junior faculty or senior faculty, or just visitors for a day or two reads like a who's who of mathematical physics. Leading the charge were Arthur Wightman, Elliott Lieb, and Barry Simon. But there were also Eugene Wigner, Valentine Bargmann, and Ed Nelson. And in applied mathematics, there was Martin Kruskal, still flush with excitement from his seminal work on the Korteweg-de Vries equation, and across the way at the Institute were Tulio Regge and Freeman Dyson, doing wonderful things. Barry was a dynamo, challenging us with open problems, understanding every lecture instantaneously, writing paper after paper, often at the seminars themselves, all the while supervising seven or eight $\mathrm{PhD}$ students.

I was one of those students. I had an appointment to meet with Barry once every two weeks. I would work very hard preparing a list of questions that I did not know how to answer. Say there were ten questions; by the end of the first ten minutes in Barry's office, the first six questions were resolved. Regarding questions seven and eight, Barry would think about them for about two or three minutes and then tell me how to do them. Regarding questions nine and ten, Barry would think about them, also for about two or three minutes, and say, "I don't know how to do them. But if you look in such and such a book or paper, you will find the answer." Invariably he was right. So in less than half an hour, all my questions were resolved, and as I walked out of the door there was the next student waiting his turn!

Barry's first $\mathrm{PhD}$ student was Tony O'Connor. O'Connor's thesis concerned exponential decay for eigenfunctions $\psi$ of $N$-body Schrödinger operators $H$, $H \psi=\lambda \psi$, for $\lambda$ below the essential spectrum of $H$. Here $H=H_{0}+V$, where $H_{0}$ is the kinetic energy and $V$ is the interaction potential. For Schrödinger operators in one dimension, such results go back to the nineteenth century, but for $N>1$ particles moving in three dimensions, completely different techniques were necessary. Over the years many people have worked on the problem, including, to name a few, Stanislav Merkuriev in the former Soviet Union, and John Morgan and Thomas and Maria Hoffmann-Ostenhof in the West. O'Connor had the idea of using the analyticity of the Fourier transform and obtained results in the $L^{2}$ sense (i.e., $e^{\alpha|\cdot|} \psi \in L^{2}, \alpha>0$ ). Such bounds are optimal for isotropic decay.

O'Connor's paper motivated Jean-Michel Combes and Larry Thomas to introduce an approach that has now become standard under the general rubric of "boost analyticity," and in a set of three papers in the mid1970s, Barry further developed these ideas to obtain pointwise exponential bounds on eigenfunctions under various assumptions on the asymptotic behavior of the interaction potential $V$, proving eventually that if $V(x)$ was bounded below by $|x|^{2 m}$, say, then one obtained superexponential decay for $\psi(x)$,

$$
|\psi(x)| \leq c e^{-\alpha|x|^{m+1}} \quad c, \alpha>0 .
$$

Schrödinger operators typically involve interaction potentials $V$ which are sums of two-body interactions

$$
V(x)=\sum_{1 \leq i<j \leq N} V_{i j}\left(x_{i}-x_{j}\right)
$$

for particles $x_{i}$ in $\mathbb{R}^{3}, i=1, \ldots, N$. Although typically

$$
V_{i j}(y) \rightarrow 0 \text { as }|y| \rightarrow \infty,
$$

$V(x)$ clearly does not decay if $|x| \rightarrow \infty$ in such a way that $x_{i}-x_{j}$, say, remains bounded for some $i \neq j$. Such nonisotropy in the potential $V$ suggests that isotropic bounds of the form

$$
|\psi(x)| \leq c e^{-\alpha|x|}
$$

are not optimal amongst all possible bounds.

In a fourth paper on exponential decay in 1978, together with Deift, Hunziker, and Vock, Barry constructed optimal nonisotropic bounds for eigenfunctions of the form

$$
|\psi(x)| \leq c e^{(\alpha, x)}
$$

for suitable $\alpha=\left(\alpha_{1}, \ldots, \alpha_{N}\right), \alpha_{i} \in \mathbb{R}^{3}$. The $\alpha$ 's reflect the geometry of the channels where

$$
V(x) \rightarrow 0 \text { as }|x| \rightarrow \infty .
$$

Eventually, in 1982, Agmon showed that for a very general class of elliptic operators $H$ in $\mathbb{R}^{3 N}$, there was a naturally associated Riemannian metric on $\mathbb{R}^{3 N}$ such that eigenfunctions $\psi$,

$$
H \psi=\lambda \psi,
$$

with $\lambda$ below the essential spectrum of $H$, satisfied the bound

$$
|\psi(x)| \leq c_{\epsilon} e^{-(1-\epsilon) \rho(x)} \quad \forall \epsilon>0,
$$

where $\rho(x)$ is the geodesic distance from $x$ to the origin in $\mathbb{R}^{3 N}$ with respect to Agmon's metric. The Agmon metric can be used to derive, and so explain, the bounds in the 1978 work of Deift, Hunziker, Simon, and Vock.

The scattering problem in quantum chemistry, going back to the 1920s, can be stated informally as follows: In a chemical reaction, do molecules go to molecules? In other words, suppose in the distant past that the particle system is described by a collection of noninteracting molecules. 


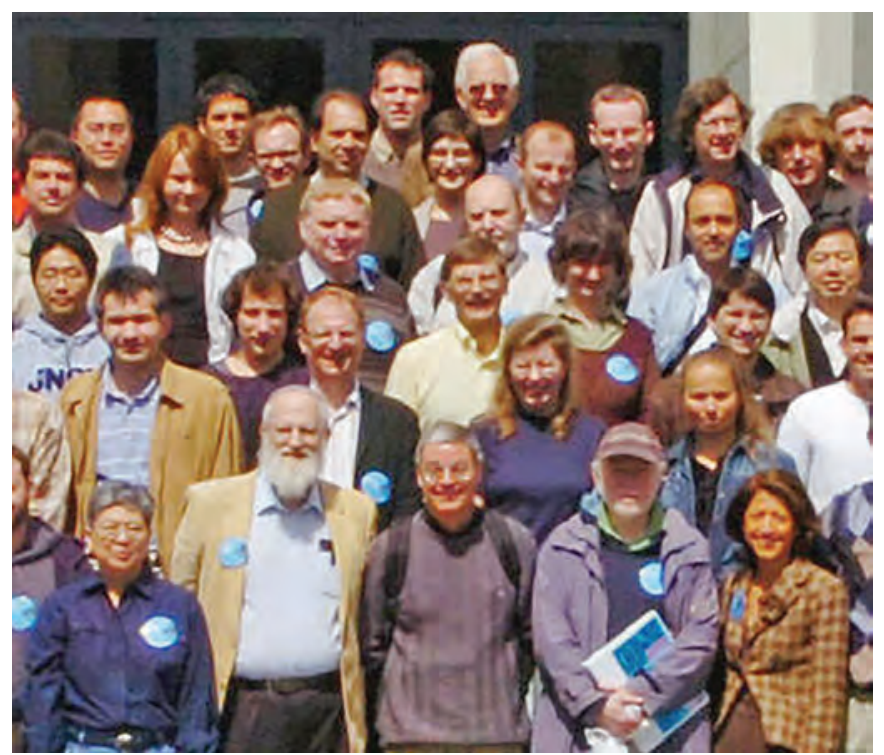

SimonFest in $\mathbf{2 0 0 6}$ for Barry Simon's sixtieth birthday. Percy Deift is in the front row, second from the right.

As time goes on, the atoms in the different molecules begin to interact with each other, and the molecules break up. At large positive times, is the particle system again described by molecules?

To be mathematically precise, consider a collection of atoms

$$
x_{i} \in \mathbb{R}^{3}, i=1, \ldots, N,
$$

with Hamiltonian

$$
H=H_{0}+V=H_{0}+\sum_{1<j \leq N} V_{i j}\left(x_{i}-x_{j}\right),
$$

in the center of mass frame. Let $C^{(1)}, \ldots, C^{(m)}$ be a decomposition of the atoms into $m$ clusters,

$$
\sum_{j=1}^{m} \#\left(C^{(j)}\right)=N \text {. }
$$

Now suppose that for large negative times the clusters $\left\{C^{(j)}\right\}$ are far apart and in each cluster $C^{(k)}$ the atoms are in an eigenstate $\psi_{k}$ of the cluster (read "molecular") Hamiltonian, that is,

$$
H^{(k)} \psi_{k}=E_{k} \psi_{k} \text {, where } H^{(k)}=H_{0}^{(k)}+V^{(k)} .
$$

Here $H_{0}^{(k)}$ is the kinetic energy for the cluster and

$$
V^{(k)}=\sum_{\substack{1 \leq i \leq j \leq \#\left(C^{(k)}\right) \\ x_{i}, x_{j} \in C^{(k)}}} V_{i j}\left(x_{i}-x_{j}\right)
$$

is the interaction potential for the atoms in the cluster. So the scattering problem of quantum chemistry becomes the following: At large positive times, is the particle system again described by molecules, that is, by some decomposition of well-separated clusters $\tilde{C}^{(1)}, \ldots, \tilde{C}^{(\tilde{m})}$ with the atoms in each cluster $\tilde{C}^{(k)}$ in an eigenstate of the cluster Hamiltonian $\tilde{H}^{(k)}$ (or more precisely, in some linear combination of such molecular configurations)?
In the physical and mathematical literature, the scattering problem is known as the problem of "asymptotic completeness" or the "unitarity of the $S$-matrix." Much work has been done on this problem by many people over the years, including the time-independent approach of Faddeev and his school, leading up to the eventual resolution of the problem in 1987 by Israel Michael Sigal and Avy Soffer using time-dependent methods pioneered by Enss.

At the mathematical level, the first task in resolving the problem is to prove that such molecular states indeed exist. This boils down to proving that the so-called wave operators $W\left(H, H_{c}\right)$ exist, where $H$ is again the Hamiltonian for the full system and $H_{c}$ is the Hamiltonian for the molecular system corresponding to the cluster decomposition $C=\left\{C^{(1)}, \ldots, C^{(m)}\right\}$. At the technical level this is a relatively easy thing to do. To prove asymptotic completeness, one must show that all states orthogonal to bound states of the full Hamiltonian $H$ are in the linear span of these molecular wave operators $W\left(H, H_{c}\right)$. This is a range question, and range questions in mathematics are generically hard.

In 1978, together with Deift, Simon introduced a
Barry is one of the most prolific mathematicians of his

\section{generation} new class of wave operators, $W\left(H_{c}, J_{c}, H\right)$, and showed that asymptotic completeness was equivalent to proving the existence of these wave operators. Here $J_{c}$ is an auxiliary function reflecting the geometry of the cluster decomposition $C$. In this way the problem of asymptotic completeness was transformed from a range problem to a potentially simpler existence problem, and this is the path that Sigal and Soffer eventually followed in their resolution of asymptotic completeness. As the ranges of the wave operators $W\left(H, H_{c}\right)$ lie in the absolutely continuous space of $H$, a key ingredient in proving asymptotic completeness was to show that the singular continuous space for $H$ was trivial. This key component was established by Barry Simon, together with Peter Perry and Israel Michael Sigal, in seminal work in 1980 using remarkable ideas of Eric Mourre [2].

\section{On a Personal Note}

Barry is one of the most prolific mathematicians of his generation. It was in the late 1970s, around the time that we were working on nonisotropic bounds for eigenfunctions, that I got a glimpse of the speed with which Barry did things. Soon after Volker Enss introduced his seminal time-dependent ideas on spectral theory and scattering theory, a few of us went to Barry's house in Edison, New Jersey, to discuss a potential project inspired by Enss's work. We spent the afternoon laying out in detail a list of problems that needed to be addressed and left in the late afternoon. The next morning Barry came 


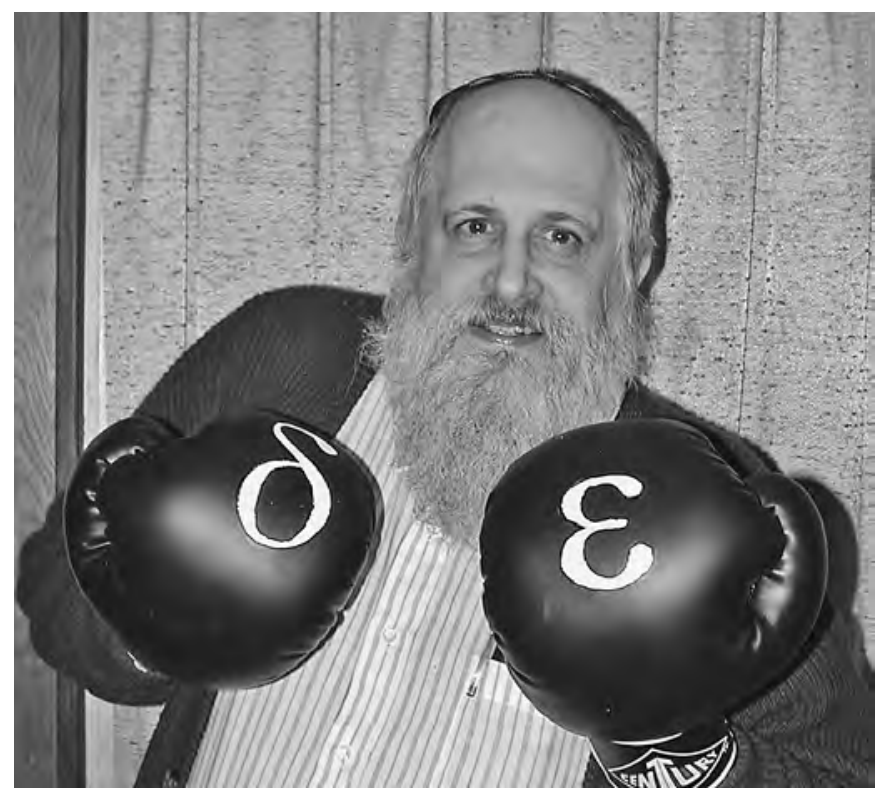

From about 1995 to 2000, Barry taught the first term of Caltech's required freshman calculus, where he declared that "epsilon and delta are a calculus student's finest weapons." One year, on the last day, the students presented him with the boxing gloves shown.

into the office: Not only had he solved all the problems on our list, but he had in his hand the first draft of his subsequent paper [3]! We were overwhelmed. For a young person like me, this was most discouraging. And I was doubly discouraged: Barry was younger than I was!

Barry has many fine qualities as a colleague and as a researcher, but I would like to focus on just one of them, viz., Barry's keen sense of fairness and correct attribution of results. People in orthogonal polynomials know well Barry's insistence on calling the recurrence coefficients for orthogonal polynomials on the circle Verblunsky coefficients, in recognition of the almost forgotten seminal work of Samuel Verblunsky. But I would like to tell a different story. In the early 1980s Barry was in Australia, where he met up with Michael Berry, who was also visiting. Berry began telling Barry about some curious and puzzling calculations he had been making in quantum adiabatic theory. Barry immediately understood that what was really going on was a matter of holonomy, and with characteristic speed he wrote and sent off a paper to Physical Review Letters, pointedly titled "Holonomy, the Quantum Adiabatic Theorem, and Berry's phase." In this way, a major discovery that could quite easily have become known as "Barry's phase" was fixed in the literature as "Berry's phase," and justly so.

\section{References}

[1] P. Deift and B. Simon, A time-dependent approach to the completeness of multiparticle quantum systems, Commun. Pure Appl. Math. 30 (1977), 573-583. MR 0459397
[2] E. Mourre, Absence of singular continuous spectrum for certain selfadjoint operators, Commun. Math. Phys. 78 (1980/81), 391-408. MR 0603501

[3] B. Simon, Phase space analysis of simple scattering systems. Extensions of some work of Enss, Duke Math. J. 46 (1979), 119-168. MR 0523604

\section{Lon Rosen}

Barry and I were both young pups when we collaborated in the 1970s. It was an intense and exciting experience for me, one which I cherish and now take pleasure in recalling for you. Warning: these personal recollections have no scientific content. If that's what you're looking for, please see my contribution to the Festschrift in honor of Barry Simon's sixtieth birthday.

I must confess that my first meeting with Barry was far from auspicious. In 1967 I was in my first year of doctoral studies at the Courant Institute. Feeling isolated, I was reconsidering my decision not to have chosen Princeton for graduate school. I asked a friend to arrange a lunch meeting for me with a typical student of Arthur Wightman's. I knew little about the "typical student" who was chosen (Barry Simon), although I was familiar with his name because Barry and I had both been Putnam Fellows in the 1965 competition.

Some typical student! He practically tore my head off. Whatever I said about my interests or ideas, Barry would trump it. I'd never met anyone else with such extensive knowledge, amazing recall, and proofs at the ready. I still haven't. Thanks to Barry, I stayed put at Courant. Fortunately, James Glimm, who was to be my terrific thesis advisor, soon joined the faculty there. I learned later that Barry had been going through a rough patch in his personal and professional life around the time we met and that the fire-breathing dragon who had me for

Lon Rosen is professsor emeritus at the Department of Mathematics, University of British Columbia. His email address is rosen@ math.ubc.ca.

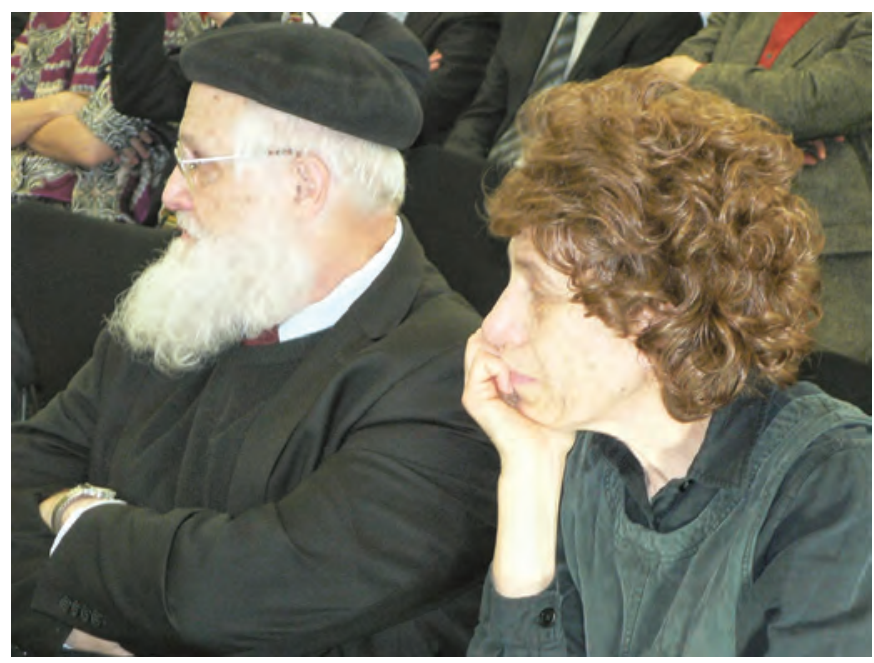

Barry and Martha Simon, LMU Munich, 2014. 
lunch was actually a gentle prince in disguise, if I may be permitted a fairy tale metaphor.

Three years later I gave a seminar at Princeton on the subject of higher-order estimates for the $P(\phi)_{2}$ model. At the conclusion of the seminar Barry showed me a clever bootstrap trick that quickly established my most difficult estimate-or at least a weaker but perfectly acceptable version of it. I was grateful and revised the published paper accordingly.

This experience was not unique to me. As many speakers know, Barry's rapid-strike ability could be unnerving at seminars. He would sit front row centre, working on a paper, only to surface with astute observations, counterexamples, or shorter proofs. This penchant for "tricks" arises, it seems to me, from Barry's imperative to understand everything in the simplest possible way.

Barry and I both attended the Les Houches Summer School of 1970. It was there that I gained an appreciation for Barry's sense of humour. In particular, we had a lot of fun putting on a skit which satirized the lecturing styles and idiosyncrasies of the various celebrated speakers. For example, when Barry began an impersonation by first breaking a half dozen chalk sticks into small pieces, everyone roared, knowing that "Arthur Jaffe" was about to deliver his next lecture.

Sometimes the humour was (possibly) unintentional. Here's a little story which Ed Nelson told me. Barry had returned from a trip to the former Soviet Union, where he had great difficulty in arranging for kosher food. It was apparently necessary for him to haul a suitcase filled with edibles. "Oh well," sighed Barry, "I guess everyone has his cross to bear."

\section{He would often come in with a twinkle in his eye}

In 1971 when I joined Barry in Princeton, we began our joint research by working on what I would call "incremental stuff using available techniques," things like coupling constant analyticity of the $P(\phi)_{2}$ Hamiltonian. One day Arthur Wightman called us together for a presentation by a quiet visitor from Italy, Francesco Guerra, whom I barely knew. Francesco proceeded to the chalkboard and made some extraordinary claims about the vacuum energy density in the $P(\phi)_{2}$ model. Barry and I gave each other a sideways look as if to say, "he's got to be kidding." He wasn't! Francesco's short proofs were stunning. The irony was not lost on us that they were based on the Euclidean approach of Ed Nelson of Princeton University. In any case, that was the moment that the Euclidean Revolution

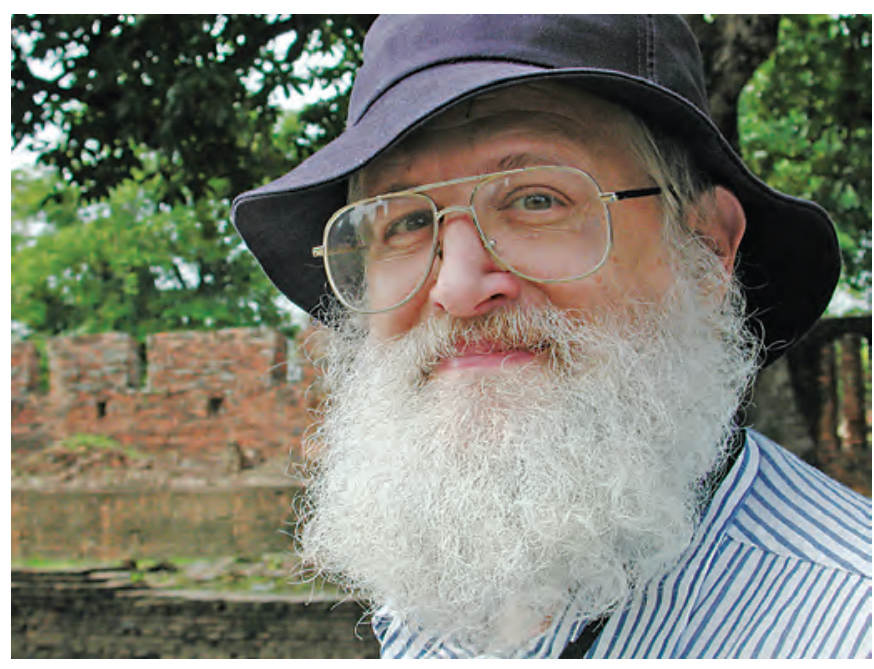

Barry Simon in Bangkok (ca. 2003).

began for Barry and me. The three of us (GRS) entered a long and fruitful collaboration exploring and exploiting the parallels between the $P(\phi)_{2}$ field theory model and classical statistical mechanics. As usual, Barry snatched up the new ideas like a dog with a bone.

During the GRS period, Barry had numerous other projects on the go, such as his research in mathematical quantum mechanics and the Reed-Simon magnum opus. Thank goodness he was devoting only a fraction of his time to GRS, whereas I was on it full time. Otherwise, I would never have been able to keep up with him and contribute my fair share. Barry's joy in doing research was infectious. He would often come in with a twinkle in his eye and say something like, "While I was standing in the supermarket line, look what I discovered!" Barry was always appreciative of my efforts and extremely generous to others. I learned a tremendous amount from him both directly during our collaboration and in subsequent years from his prodigious published output.

\section{Jürg Fröhlich}

\section{Barry Simon and Statistical Mechanics}

Well, this is about my mentor and friend Barry Simon! I first met Barry at a summer school on constructive quantum field theory and statistical mechanics at Les Houches, France, in 1970. We were only twenty-four years old at the time, and I had just started my life as a PhD student of Klaus Hepp, while Barry, a former PhD student of the late Arthur S. Wightman, was already a "Herr Doktor" and-if my memory is correct-an assistant professor at Princeton University. As I wrote on the occasion of his sixtieth birthday celebrations [1], Barry would usually beat me in almost everything! To start with, he was born two and a half months before me.

At Les Houches, the late Oscar E. Lanford III lectured on general functional analysis, including measure theory

Jürg Fröhlich is professor emeritus of the Department of Physics at the ETH, Zürich. His email address is juerg@phys. ethz.ch. 
and the theory of operator algebras, tools rightly thought to be essential to understanding quantum theory and statistical mechanics. Besides Barry and me, Alain Connes was a student at that school. Barry and Alain were soon engaged in a competition to simplify Oscar's proofs; Barry would usually win-not only in mathematics but also in the consumption of food.

Our common mentor and friend, the late Edward Nelson, wrote about Barry [1]: "In the late 1960s, Barry was a graduate student in physics at Princeton and attended some courses I taught. I soon learned that I did not need to prepare with great thoroughness; it was enough to get things approximately right and Barry from where he was sitting would tell us how to get them precisely right. I miss Barry.” Well, Barry and I miss Ed!

My next encounter with Barry was in 1972 when he taught a graduate course on quantum field theory in the "3ième cycle" of French-speaking Switzerland. That course was the basis for his $P(\phi)_{2}$-book, which is still remembered in the community. This is perhaps because it contains those famous "Fröhlich bounds" or, more likely, because it is written in a very pedagogical mannerindeed, one of Barry's outstanding strengths was and still is to being able to write mathematical prose in a very clear, pedagogical style.

In the fall of 1974 I accepted the offer of an assistant professorship at the mathematics department of Princeton University which had been prepared by Arthur Wightman and Barry Simon. Major benefits of having had Barry as a colleague were that I never had to submit a grant proposal to the NSF; Barry's proposal not only covered his own needs but also the ones of the late Valja Bargmann and me, and should one successfully collaborate with him, he would always write the paper (except for appendices on tedious technicalities, such as cluster expansions, which he gracefully assigned to his collaborators).

\section{Barry needed only roughly 5 percent of the time ordinary mortals need to write a} paper

Barry needed only roughly 5 percent of the time ordinary mortals need to write a paper. He did his writing while listening to seminar talks. Although he appeared to be absorbed in his activity (carried out by moving his left hand along rather peculiar trajectories), he would nevertheless be able to point out errors to the lecturer or ask relevant questions at the end. Barry was simply brilliant in "multitasking."

Let me briefly describe two of our joint papers. The first one is entitled "Pure states for general $P(\phi)_{2}$-theories: Construction, regularity and variational equality" and was published in the Annals of Mathematics in 1977. In this paper, ideas and concepts from classical statistical mechanics were transferred to Euclidean field theory with the purpose of learning something new about the latter. General concepts were illustrated on simple examples of Euclidean field theory in two dimensions, which, mathematically, may be defined as generalized stochastic processes-more precisely, Markovian random fields-over $\mathbb{R}^{2}$ constructed as perturbations of Gaussian processes by local multiplicative functionals. From such processes interacting scalar quantum field theories on two-dimensional Minkowski space can be reconstructed, furnishing examples of what has become known as constructive quantum field theory. CQFT was first advocated by Arthur Wightman in the early 1960s with the purpose of showing that quantum theory and the special theory of relativity are compatible with each other, and was subsequently pursued by Edward Nelson, James Glimm, Arthur Jaffe, and their followers.

The modern mathematically rigorous approach to statistical mechanics was developed by, among many other people, Roland L. Dobrushin, Oscar E. Lanford, and, most importantly, David Ruelle. Our paper is unthinkable without their work and without the discoveries of K. Symanzik, E. Nelson, F. Guerra, L. Rosen, and B. Simon in Euclidean field theory, some of whose works are classics. They had shown that in the Euclidean region (time purely imaginary) of complexified Minkowski space, a quantum field theory of Bose fields looks like a model of classical statistical mechanics. In my paper with Barry this fact is exploited in an essential way.

We were to "win the jackpot" with the paper "Infrared bounds, phase transitions and continuous symmetry breaking," which was the result of joint work with our friend Tom Spencer and was published in Communications in Mathematical Physics in $1976 .{ }^{1}$ Barry, Tom, and I decided to attempt to understand phase transitions accompanied by the spontaneous breaking of continuous symmetries and long-range correlations (i.e., a divergent correlation length), in models of classical lattice spin systems and lattice gases. We exploited ideas from quantum field theory; in particular, we discovered an analogue of the so-called Källen-Lehmann spectral representation of two-point correlation functions in quantum field theory. For this representation to hold true it is necessary that the model under scrutiny satisfy the Osterwalder-Schrader positivity, also called reflection positivity, a property originating in axiomatic quantum field theory (as described in well-known books by Streater and Wightman and by Jost).

Here is an example: With each site $x$ of the lattice $\mathbb{Z}^{d}$ we associate a random variable $\vec{S}_{x} \in \mathbb{R}^{N}$, a classical "spin," whose a priori distribution is given by a probability measure, $d \mu(\cdot)$ on $\mathbb{R}^{N}$, invariant under rotations of $\mathbb{R}^{N}$; for instance,

$$
d \mu(\vec{S})=\text { const. } \delta\left(|\vec{S}|^{2}-1\right) d^{N} S .
$$

Let $\Lambda$ be a finite cube in $\mathbb{Z}^{d}$. The energy of a configuration, $\vec{S}_{\Lambda}:=\left\{\vec{S}_{X}\right\}_{X \in \Lambda}$, of "spins" is given by a functional (called "Hamiltonian"),

$$
H\left(\vec{S}_{\Lambda}\right):=-\sum_{x, y \in \Lambda} J(x-y) \vec{S}_{x} \cdot \vec{S}_{y} .
$$

\footnotetext{
${ }^{1}$ At the beginning of my career I was privileged to have several mentors, among whom Tom was undoubtedly the most important one!
} 


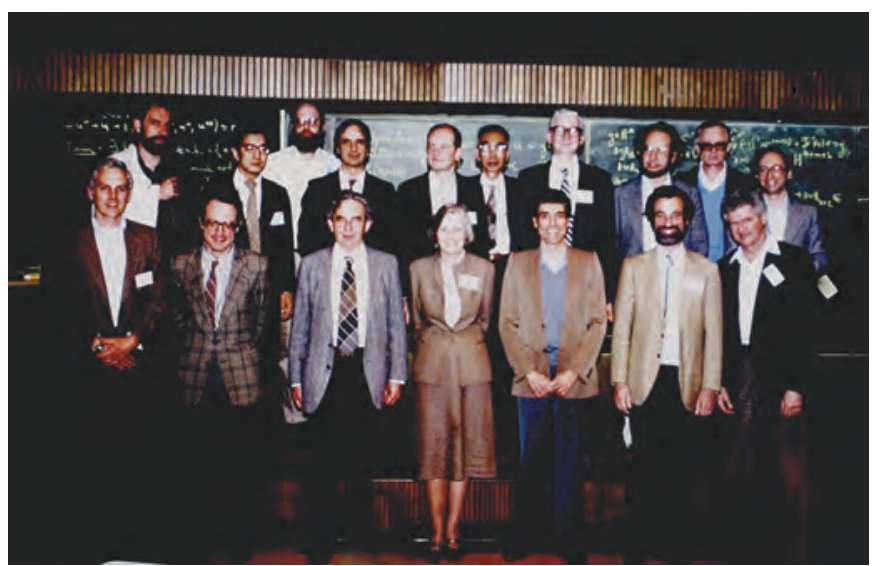

Birmingham, AL, Meeting on Differential Equations, 1983. Back row: Fröhlich, Yajima, Simon, Temam, Enss, Kato, Schechter, Brezis, Carroll, Rabinowitz. Front row: Crandall, Ekeland, Agmon, Morawetz, Smoller, Lieb, Lax.

Here $J(x)$ is a function in $\ell_{1}\left(\mathbb{Z}^{d}\right)$ assumed to be reflectionpositive and invariant under permutations of lattice directions. By a theorem of Bochner, these properties imply that it has an integral representation,

$$
J\left(x_{1}, \vec{x}\right)=\int_{-1}^{1} \lambda^{\left|x_{1}\right|-1} e^{i \vec{k} \cdot \vec{x}} d \rho(\lambda, \vec{k}), \quad x_{1} \neq 0,
$$

with $\vec{x}=\left(x_{2}, \ldots, x_{d}\right)$, where $d \rho(\lambda, \vec{k})$ is a positive measure on $[-1,1] \times \mathbb{T}^{d-1}$, for example, $J(x)=\delta_{|x|, 1}$. In (5) we

impose periodic boundary conditions at the boundary of $\Lambda$. The distribution of configurations $\vec{S}_{\Lambda}$ of "spins" in thermal equilibrium at inverse temperature $\beta$ is given by the Gibbs measure

$$
d P_{\beta}\left(\vec{S}_{\Lambda}\right):=Z_{\beta, \Lambda}^{-1} \exp \left[-\beta H\left(\vec{S}_{\Lambda}\right)\right] \prod_{x \in \Lambda} d \mu\left(\vec{S}_{x}\right),
$$

where $Z_{\beta, \Lambda}$ is a normalization factor (called "partition function"). Let $\langle\cdot\rangle_{\beta, \Lambda}$ denote an expectation with respect to $d P_{\beta}$. For "wave vectors" $k$ in the lattice dual to $\Lambda$, we define $\omega_{\beta, \Lambda}(k)$ to be the Fourier transform of the correlation function $\left\langle\vec{S}_{0} \cdot \vec{S}_{x}\right\rangle_{\beta, \Lambda}, 0, x \in \Lambda$.

Simon, Spencer, and I proved that

$$
0 \leq \omega_{\beta, \Lambda}(k) \leq \frac{N}{2 \beta(\hat{J}(0)-\hat{J}(k))} \text { for } k \neq 0, \forall \beta,
$$

where $\hat{J}(k)$ is the Fourier transform of $J(x), x \in \Lambda$. This socalled "infrared bound" is inspired by the Källen-Lehmann representation of two-point functions in canonical relativistic quantum field theory. The realization that (6) implies the upper bound in (8) is the basic result in our work. It is then an exercise to show that if the "coupling function" $J(\cdot)$ is such that

$$
|\Lambda|^{-1} \sum_{k \neq 0}[\hat{J}(0)-\hat{J}(k)]^{-1} \leq \text { const., }
$$

uniformly in $\Lambda$, then in the thermodynamic limit $\Lambda \nearrow \mathbb{Z}^{d}$, phases with broken $O(N)$-symmetry coexist and are permuted among themselves under the action of the symmetry group $O(N)$, provided $\beta$ is large enough. (By (4), $\left\langle\left|\vec{S}_{0}\right|^{2}\right\rangle_{\beta, \Lambda}=1$; this and (8), (9) imply that the weight of the mode at $k=0$ in $\omega_{\beta, \Lambda}(k)$ is $\propto|\Lambda|$.) For small $\beta$, however, the Gibbs state is well known to be unique.

It turns out that the bound (8) has many further applications. It is an important ingredient in a beautiful analysis of critical behavior in the Ising model (by Aizenman, Duminil-Copin, and Sidoravicius) and in showing that the large-distance scaling limit of the nearest-neighbor Ising and the classical XY-model is Gaussian in dimension $d>4$ ("triviality of $\lambda \phi_{d}^{4}$-theory" in $d \geq 4$ dimensions); see [2], [3].

In 1980 Barry proved a correlation inequality, sometimes referred to as the "Simon-Lieb inequality," useful to establish decay of correlations in models of classical ferromagnetic lattice spin systems, e.g., the one sketched above with $N=1$ or 2 . Two years later, the basic idea expressed in his inequality became a very useful ingredient in the analysis of multiscale problems, such as Anderson localization (as in work by Tom Spencer and me).

To conclude, let me draw the reader's attention to Barry Simon's book The Statistical Mechanics of Lattice Gases.

\section{References}

[1] math.ca1tech.edu/SimonFest/stories.htm7

[2] M. Aizenman, Geometric analysis of $\phi^{4}$-fields and Ising models, Commun. Math. Phys. 86 (1982), 1-48.

[3] J. Fröhlich, On the triviality of $\lambda \phi_{d}^{4}$-theories and the approach to the critical point in $d \geq 4$ dimensions, Nucl. Phys. B 200 (1982), 281-296.

\section{Mike Reed}

\section{On Barry Simon}

When people ask, "How long did it take for you and Barry Simon to write those four volumes of Methods of Modern Mathematical Physics?" I usually say, "About ten years," since we started in the late 1960s when Barry was a graduate student and I was a lecturer at Princeton, and we finished in the late 1970s. Writing those books took 50 percent of my research time for ten years but only 10 percent of Barry's research time, and that wasn't because I contributed more-far from it. The reason is that no one works faster than Barry. He instantly sees the the significance of new ideas (whether in mathematics or physics), understands the technical structures necessary to bring the ideas to fruition, and immediately starts writing.

Barry's legendary speed sometimes got him into trouble. I remember going to seminars at Princeton with Barry carrying new preprints from more senior mathematicians. As the seminar proceeded, Barry would read the preprint, absorb the idea, understand the correct machinery to prove a stronger result, and begin writing. No one is more generous than Barry at giving credit to others; he always does and did. Nevertheless, when Barry's paper with a stronger result and a better proof would appear before

Mike Reed is professor and Bass Fellow in Mathematics at Duke University. His email address is reed@math. duke.edu. 


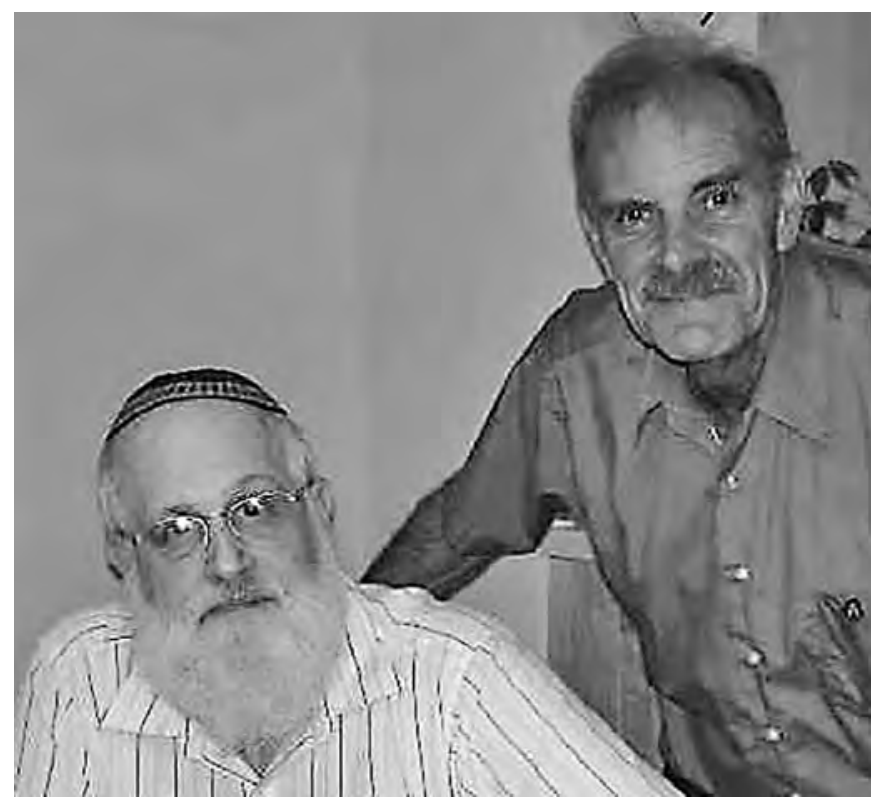

Barry Simon and Michael Reed, Durham, NC, 2007.

the original result, the preprint's author would sometimes have hard feelings. These feelings would usually dissipate when he or she actually met Barry and discovered how open and generous he is.

We wrote the books because we saw that the physics of quantum mechanics and quantum field theory raised deep and interesting analysis questions. Of course our ancestors knew this too. But we saw how hard it was for mathematicians to understand the issues and fully engage because it was so difficult to read the physics literature and translate the ideas and computations into mathematical questions. Originally, we were going to write one small volume that would give the functional analysis background followed by short chapters introducing mathematicians to problems in modern physics. But we were driven by Barry's deep knowledge and intuition about physics and our shared enthusiasm to do and say more, and the result is the four volumes that we wrote.

We had a terrific time! This was long ago, so our handwritten manuscripts were typed. Then we would take the typed manuscript, usually 500-700 pages, and read it aloud. This was the only way to go slowly enough to check the English, the mathematics, and the physics. One would read, both would think, and the other would write down corrections. Typically it took three weeks full time to read a manuscript. For a couple of them, I lived with Barry. We'd get up in the morning, get He respects others, to work, and give up when we were tired in whatever their talent, whatever their station in life the evening. We were blessed by the tolerance and good cooking of Barry's wife, Martha. It was very rare that we'd be irritated or angry at each other, because we both have strong personalities that are not easily troubled and we were completely focused on the mathematics and the science. We did all the problems in all the volumes, except the starred ones that we sure were correct but couldn't immediately see how to do.

Of course, we were pleased and proud that so many colleagues and students found our books useful. We both still teach out of them and field email questions about the problems. Since we were so young when they were written, we got lots of funny remarks at conferences from mathematicians who didn't know us, such as, "You can't be the Simon who wrote those books; you're too young," and, "Hah! I always thought that Reed was Simon's first name."

There are lots of things to celebrate about Barry Simon: his stupendous achievements, his many students, his sense of humor, his generosity to colleagues. I celebrate his deep sense of common humanity with other human beings. He respects others, whatever their talent, whatever their station in life, and this sense of common humanity makes him very special.

This article will continue next month with contributions by S. Jitomirskaya, Y. Avron, D. Damanik, J. Breuer, Y. Last, and A. Martinez-Finkelshtein. 
Barry Simon's Students at Princeton

Anthony O'Connor, 1972

Jay Rosen, 1974

Robert Israel, 1975

Percy Deift, 1976

Evans Harrell II, 1976

George Hagedorn, 1978

Mark Ashbaugh, 1980

Antti Kupiainen, 1980

Steven Levin, 1980

Peter Perry, 1981

Keith Miller, 1982

Barry Simon's Students at the California Institute

of Technology

Byron Siu, 1984

Nestor Caticha Alfonso, 1985

Barton Huxtable, 1987

Kristiana Odencrantz, 1987

Clemens Glaffig, 1988

Askell Hardarson, 1988

John Lindner, 1989

Vojkan Jaksic, 1992

Yunfeng Zhu, 1996

Alexander Kiselev, 1997

Andrei Khodakovsky, 1999

Rowan Killip, 2000

Andrej Zlatos, 2003

Irina Nenciu, 2005

Mihai Stoiciu, 2005

Manwah Wong, 2009

Rostyslav Kozhan, 2010

Anna Maltsev, 2010

Milivoje Lukic, 2011

Brian Zachary Simanek, 2012

\section{Credits}

p. 740 Childhood photo of Simon with family, courtesy of Barry Simon.

p. 741 Photo of Simon receiving his honorary doctorate, photographer Heinrich Steinlein. Used with permission.

p. 742 Photo of Simon's books, courtesy of Barry Simon.

p. 743 Photo of Sadun, Avron, Harrell, and Simon, courtesy of Evans Harrell.

p. 743. The Stark Effect, used with permission of The American Physical Society.

p. 744 Photo of Simon's coauthors, courtesy of The California Institute of Technology.

p. 745 Photo of Simon's students, courtesy of The California Institute of Technology.

p. 746 Photo of 2006 SimonFest, courtesy of The California Institute of Technology.

p. 747 Photo of Simon in boxing gloves, courtesy of Barry Simon.

p. 747 Photo of Barry and Martha Simon, photographer Heinrich Steinlein. Used with permission.

p. 748 Photo of Simon in Bangkok, courtesy of Barry Simon.

p. 750 Photo of Birmingham Meeting on Differential Equations, courtesy of Barry Simon.

p. 751 Photo of Simon and Reed, courtesy of Barry Simon. 